\title{
DNA-Liposome Hybrid Carriers for Triggered Cargo
}

\section{Release}

Kevin N. Baumann ${ }^{1,2 \dagger}$, Tim Schröder ${ }^{3}$, Prashanth S. Ciryam ${ }^{1 *}$, Diana Morzy ${ }^{2 \S}$, Philip Tinnefeld ${ }^{3,4}$,

Tuomas P. J. Knowles ${ }^{1,2 *}$, Silvia Hernández-Ainsa ${ }^{5,6 *}$

\section{AUTHOR ADDRESSES}

1 Yusuf Hamied Department of Chemistry, University of Cambridge, Lensfield Road, Cambridge CB2 1EW, United Kingdom

2 Cavendish Laboratory, University of Cambridge, JJ Thomson Avenue, Cambridge CB3 0HE, United Kingdom

3 Department of Chemistry, Ludwig Maximilian University München, Butenandtstr. 5 - 13, 81377 Munich, Germany

4 Center for NanoScience, Ludwig Maximilian University München, Geschwister-Scholl Platz 1, 80539 Munich, Germany

5 Instituto de Nanociencia y Materiales de Aragón, CSIC-Universidad de Zaragoza, Zaragoza 50009, Spain

6 ARAID Foundation, Government of Aragon, Zaragoza 50018, Spain 
KEYWORDS DNA nanotechnology; biomimetics; liposome; triggered release; drug delivery

ABSTRACT The encapsulation of chemotherapeutics by biocompatible carrier structures holds great promise to preserve their therapeutic activity and favor their delivery to tumor sites. To enhance the bioavailability of a drug at the targeted tissue, triggered release mechanisms have received increasing research interest. Many approaches rely on exogeneous triggers such as the irradiation of ultrasound, visible or even ionizing electromagnetic waves. However, such exogenous triggers can be challenging to implement in a specific manner. Therefore, designing carriers responsive to endogenous moieties, such as nucleic acid biomarkers, is a desirable step in the search of personalized drug delivery nanoplatforms. This study presents an approach to building a biocompatible DNA-liposome hybrid nanocarrier for potential triggered release purposes. We form a DNA mesh on large unilamellar liposomes incorporating a trigger-responsive DNA building block. Upon incubation with a single-stranded DNA trigger sequence a hairpin closes and the building block is allowed to self-contract. By this process, we demonstrate elevated release of the dye calcein and the drug doxorubicin. The incubation of the doxorubicin-laden active hybrid carrier with HEK293T cells suggests increased cytotoxicity relative to a control carrier without the triggered release mechanism. In the future, the trigger could be provided by peritumoral nucleic acid sequences and lead to site-selective release of encapsulated chemotherapeutics. 
TEXT

Treatment with many drugs, especially chemotherapeutics, can be associated with severe side effects. Following administration, drug molecules can circulate throughout the blood stream and can be internalized by a range of cells depending on their rate of metabolism - regardless of whether they are of cancerous or of healthy origin. ${ }^{1,2}$ Another limitation is that small molecules are generally cleared out of the organism rapidly. To address these challenges, the development of larger carrier constructs has shown promise, especially if these are targeted. ${ }^{3-6}$ Thus, the encapsulation of small therapeutic molecules by carrier structures holds great potential to maximize delivery efficiency. To fulfil a therapeutic effect, however, the drug must become bioavailable by being released from the carrier. ${ }^{7-9}$ This requires the precise control over the mechanism and timing of cargo release. ${ }^{7}$

Exogenous triggers, such as electromagnetic radiation, allow the precise timing of trigger deployment, but in many cases require additional intrusion and interference with the organism, or the use of highly specialized materials. Moreover, the irradiation of e.g. ultraviolet or visible light as trigger types is hampered by the shallow penetration depths in biological tissues..$^{10-12} \mathrm{X}$-rays in particular offer greater penetration and can be used in combination with radiotherapy.,13 In other applications, however, ionizing radiation imposes an additional risk. For these reasons, an entirely autonomous device with release properties dependent on an endogenous trigger event may be a preferrable solution. ${ }^{14}$

DNA as a building material has great potential to create a drug delivery vehicle responding to endogenous triggers. ${ }^{15-18}$ The triggered hybridization of DNA can produce forces large enough to facilitate the transition between secondary structures. ${ }^{19-21}$ It has been previously shown that the 
binding of an aptamer sequence to its target structure can displace pre-hybridized complementary DNA from the aptamer. ${ }^{16}$ This approach has the advantage that an overexpressed protein can facilitate targeted delivery and selective drug release at the same time. Interestingly, also nucleic acids can be overexpressed by tumor cells and be present at elevated concentrations in the peritumoral environment. ${ }^{22-24}$ The hybridization of these nucleic acids with a corresponding carrier-associated DNA motif could therefore initiate the conversion between secondary structures and thus trigger drug release by direct mechanical interference with the delivery vehicle, such as a lipid vesicle.

Lipid vesicles represent a biocompatible carrier structure that can be produced with high throughput. ${ }^{25,26} \mathrm{DNA}$ can be easily anchored to the lipid bilayer mediated by chemically attached hydrophobic moieties. ${ }^{27-31}$ Several studies demonstrate a strong link between the conformation of the DNA structures and the shape or integrity of the lipid bilayer. ${ }^{27,32,33}$ However, many current approaches rely on stochastic modes of deforming lipid vesicles. Triggering the structural activation of liposome-associated DNA building blocks to affect the permeability of the lipid bilayer to specific molecules could offer a potent strategy of generating a drug delivery vehicle with a selective release feature.

This study presents a method to release small molecules from liposomes by the triggered contraction of 'active DNA building blocks' (aDBB). These are arranged on the surface of the liposomes and integrated into a DNA coat, following a modified assembly method of an approach which we described previously. ${ }^{34}$ The addressability of the aDBB leverages the triggered selfhybridization of a DNA hairpin $(\mathrm{H})$, resulting in a contraction of the aDBB. To instate control over the hairpin closure, a spacer strand (S) was pre-annealed with the hairpin to keep it in an open, or stand-by state (Figure 1a). When adding a trigger sequence (C), S can be displaced in a toehold- 
mediated reaction. The HS DNA duplex (aDBB) was inserted between a cholesterol-triethylene glycol (TEG)-modified linker ( $\mathrm{L}$ composed of $\mathrm{L}_{1}$ and $\mathrm{L}_{2}$, Figure 1a) and a DNA triskelion (T) by hybridization via the oligonucleotides $\mathrm{L}_{2}$ (part of the linker and partially complementary to $\mathrm{H}$ ) and M (linking $\mathrm{H}$ to $\mathrm{T}$ ). The cholesterol-TEG modification of $\mathrm{L}$ allowed the aDBB to insert into lipid membranes (Figure 1b). In this manner, several of these motifs could be connected on the surface of liposomes in a two-step polymerization process: first, the combination of the aDBB, the cholesterol-TEG-labelled $\mathrm{L}$, and $\mathrm{M}$ (referred to as $\mathrm{L}_{\mathrm{HS}}$ ) was incubated with large unilamellar vesicles (LUVs). Second, the DNA triskelion was added to finalize the coating process (Figure 1c). The element-wise contraction of the DNA building blocks integrated in the DNA coat could alter the permeability of the assembled structures and facilitate the release of encapsulated molecules.

To probe the ability of the active building block to contract upon stimulation with the trigger sequence C (Figure 1a), S was modified with a Cy3 fluorophore (pink sphere, Figure 2a), whereas C was modified with a BlackHole II quencher (black sphere) in the complementary position (Figure 2a). Figure 2 shows the fluorescence signal decrease as a result of the hybridization of $\mathrm{C}$ and $\mathrm{S}$, indicating the success of the displacement reaction. As a reference, a control sequence $\mathrm{C}_{\mathrm{C}}$ was added (blue data trace). Here, only an insignificant drop of the fluorescence intensity due to stochastic quenching could be observed, comparable to the effect of dilution as simulated by adding pure PBS (grey data trace). Single-molecule FRET measurements were performed to measure the inter-dye distance, quantified by the FRET efficiency, upon contraction of the aDBB. To this end, the DNA strands adjacent to $\mathrm{H}$ were labelled with a Cy3 fluorophore (pink sphere) as donor molecule (strand $\mathrm{L}_{2}$, Figure 2c), and a Cy5 fluorophore (red sphere) as acceptor molecule (strand M, Figure 2c). Before addition of C, the FRET efficiency was zero (Figure 2c) which is 
reasonable for an estimated donor acceptor distance of approximately $15 \mathrm{~nm}$ (see Supporting Information for details) and a Förster-radius of ca. $5.3 \mathrm{~nm} .{ }^{35}$ After the displacement of S, however, the FRET efficiency shifted to around 45\%, which accounts for a distance of approximately $6 \mathrm{~nm}$ for this donor-acceptor pair (using Gamma-corrected values, see also experimental section). ${ }^{35-37}$ This finding suggests that the displacement of $\mathrm{S}$ upon hybridization with $\mathrm{C}$ indeed causes a contraction of up to ca. $9 \mathrm{~nm}$ of the building block by allowing $\mathrm{H}$ to self-hybridize.

To confirm the desired integration of the aDBB in the formerly established DNA coat, dynamic light scattering and $\zeta$-potential measurements were performed. An increase of the hydrodynamic diameter was observed, accompanied by a decrease of the $\zeta$-potential, when the DNA coats are assembled on the vesicle surfaces (Supporting Information, Figure S2). Furthermore, the addition of a detergent led to a double peak in the DLS spectrum denoting detergent-lipid micelles and coexisting DNA assemblies, as we previously described ${ }^{34}$ Next, we sought to gain further insight of the functionality of the aDBB when integrated in the DNA coats on liposomes. To this end, we studied DNA-coated giant unilamellar vesicles (GUVs) by confocal microscopy and fluorescence recovery after photobleaching (FRAP) measurements. Initially, the fluorescence recovery profiles of the ATTO550-labelled S and the ATTO647N-labelled T were measured before adding the trigger sequence $\mathrm{C}$. The two species showed similar recovery kinetics and levels of recovered fluorescence (Figure 3a and c). In comparison to our previously established DNA coats without the aDBB, here a construct with higher mobility and fewer constraints is present. This may be related to lower polymerization efficiency or to the introduction of more degrees of freedom by structures of larger flexibility (additional sites of ssDNA as knickpoints, refer to Figure $1 \mathrm{a}) .{ }^{34}$ After adding $\mathrm{C}$, the recovery traces of $\mathrm{S}$ and $\mathrm{T}$ diverged (Figure $3 \mathrm{~b}$ ) and two populations of recovery behaviors emerge: while $\mathrm{S}$ shows faster recovery kinetics and higher recovered fluorescence 
intensity levels (Figure 3c and d), the recovery of $\mathrm{T}$ is represented by slower kinetics and only marginal recovered fluorescence intensity (Figure $3 \mathrm{e}$ and f). This result corroborates the hypothesis that $\mathrm{S}$ is removed by $\mathrm{C}$, which can consequently dissociate from the DNA coat and hence is not restraint in its mobility by the DNA coat anymore. These results suggest that the transition of the SC duplex into the solution is limited by steric hindrance imposed by the DNA coat, and magnesium-assisted unspecific adsorption to the lipid bilayer, which allowed the observation by FRAP with overall reduced signal intensity (relative to the ATTO647N-labelled $\mathrm{T}$ by approximately 50\%, see also Supporting Information, Figure S3). ${ }^{38}$ At the same time, the hairpin can self-hybridize and may stiffen or compact the DNA coat, as indicated by the slow recovery kinetics of $\mathrm{T}$ and the reduced post-bleaching fluorescence intensities which exhibit similar properties to the DNA coats we previously described ${ }^{34}$ During this process, we could not detect visible deformation or disruption of the GUVs (Supporting Information, Figure S3). By contrast, when the displacement reaction is performed under hyperosmotic pressure, membrane budding or tubulation can be observed in some cases (Figure 3g).

As an indicator of the applicability of the DNA-liposome hybrid structures as triggered-release delivery vehicles, we investigated potential changes of the permeability to entrapped small molecules. Therefore, the lipid films were rehydrated in a calcein-containing solution to generate calcein-laden LUVs. The leakage of calcein from the LUVs is expressed by an increase of the fluorescence intensity, as the consequent dilution of the fluorophore results in a loss of selfquenching which only occurs at high concentrations. Initially, we measured the passive leakage of calcein from pure liposomes $(\mathrm{V})$ in comparison to the coated structures $\mathrm{VL}_{\mathrm{HS}} \mathrm{T}$ and VLT. The addition of the DNA coats, significantly reduced the leakage of calcein over the course of the measurement. To study the active release, the trigger sequence $\mathrm{C}$ was added to the samples $\mathrm{V}$, 
$\mathrm{VL}_{\mathrm{HS}} \mathrm{T}$ and VLT (as inactive control of the hybrid carriers). In contrast to VLT, the triggerresponsive carrier $\left(\mathrm{VL}_{\mathrm{HS}} \mathrm{T}\right)$ responded to the supplementation of $\mathrm{C}$ by an elevated permeability to the entrapped calcein, close to pure POPC liposomes (Figure 4a). In addition, we performed a control experiment where an inactive sequence $C_{C}$ was incubated with $\mathrm{VL}_{\mathrm{HS}} \mathrm{T} . \mathrm{C}_{\mathrm{C}}$ did not cause a similar permeability benefit, excluding the possibility that the release was only an effect of osmotic changes.

To further explore controlled drug delivery and release applications, we produced LUVs that encapsulated the widely-used chemotherapeutic doxorubicin (DOX). ${ }^{39-41}$ As a model culture, HEK293T cells were then incubated with DOX-laden $\mathrm{VL}_{\mathrm{HS}} \mathrm{T}$, and VLT. The trigger strand C was added to the DOX-laden carriers, whereby only the $\mathrm{VL}_{\mathrm{HS}} \mathrm{T}$ design is expected to respond to the trigger and increase the permeability to DOX. After the incubation time, the cytotoxic effect of the two carriers was estimated using a luciferase viability assay. Figure $4 \mathrm{~b}$ shows that upon addition of $\mathrm{C}$ the toxicity of the trigger-responsive carrier design $\left(\mathrm{VL}_{\mathrm{HS}} \mathrm{T}\right)$ is significantly increased with respect to the non-responsive VLT design which we assigned to the enhanced permeability of the vesicle promoted by the activation of the aDBB.

In conclusion, in this study we demonstrate an approach to add release functionality to DNAcoated vesicles. We provide evidence that the triggered closure of a DNA hairpin can influence the DNA coat and lipid membrane properties. This effect is likely to originate from the contraction of the hairpin following its self-hybridization. This strategy can be applied to triggered release purposes, which we evidence by the release of dye molecules and the enhanced cytotoxicity induced by the DOX-laden trigger-responsive coated liposomes. In the future, the presented method may inspire similar approaches with maximized control of the deformation of liposomes. We believe that a system like this can have significant potential for nanotherapeutic applications 
as it allows molecules to be transported and released only when a trigger is present. This could be exploited for instance in the proximity of tumors, where the bioavailability of enclosed molecules can be increased by triggering the release through the interaction with pathophysiologically overexpressed biomolecules and the carriers..$^{3,42-46}$

\section{Method Section}

\section{Folding of the Active DNA Building Block}

The aDBB was folded in two steps. First the strands $\mathrm{S}$ and $\mathrm{H}$ were hybridized using a custom thermal protocol: in 1x PBS $\left(\mathrm{pH}=7.4\right.$, or an aqueous solution $75 \mathrm{mM} \mathrm{Na}_{2} \mathrm{HPO}_{4}, \mathrm{pH}=7.4$ for the leakage measurements) $6 \mu \mathrm{M}$ of the two oligonucleotides was suspended and heated to $85{ }^{\circ} \mathrm{C}$ for $5 \mathrm{~min}$, before cooling to room temperature at a rate of $-0.5^{\circ} \mathrm{C}$ per minute. Eventually, the samples were kept at $4{ }^{\circ} \mathrm{C}$. In the second step, the S-H-duplex was incubated with L (separately annealed following our previously published protocol $^{34}$ ) and the strand M. All oligonucleotides were purchased from Integrated DNA Technologies (IDT). The sequences of all involved oligonucleotide sequences can be reviewed in the Supporting Information, Table S1.

\section{Gel Electrophoresis}

Polyacrylamide gel-electrophoresis (PAGE) was performed to evaluate the folding success the DNA structures. The gels were prepared with $10 \%$ polyacrylamide in $11 \mathrm{mM} \mathrm{MgCl}$ buffered at $\mathrm{pH}=8.3$ with $0.5 \mathrm{x} \mathrm{TBE}$ and run for $60 \mathrm{~min}$ at $100 \mathrm{~V}$, immersed in a solution containing $11 \mathrm{mM}$ $\mathrm{MgCl}_{2}$ buffered at $\mathrm{pH}=8.3$ with $0.5 \mathrm{x}$ TBE. 


\section{Fluorescence quenching measurements}

The efficacy of the toehold-mediated displacement reaction in removing the spacer strand was further verified by measuring fluorescence quenching when hybridizing with the displacement strand. To this end, $\mathrm{C}$ was labelled with an Iowa Black-quencher in the 5'-terminal and $\mathrm{S}$ with a Cy3 fluorophore in the 3'-terminal (purchased from IDT). The degree of fluorescence quenching upon addition of the displacement strand correlates with the amount of displaced spacer. The $\mathrm{aDBB}$ was studied at a concentration of $2 \mu \mathrm{M}$; the displacement strand was added at $2 \mathrm{x}$ excess (to increase the displacement success, following PAGE results). As a control, a non-hybridizing sequence $\mathrm{C}_{\mathrm{C}}$ was labelled with an Iowa Black-quencher and added under the same concentration conditions. As a further control, PBS was added at the same volume as $\mathrm{C}_{\mathrm{C}}$ to compare the stochastic quenching originating from the addition of $\mathrm{C}_{\mathrm{C}}$ to the fluorescence reduction originating from dilution.

\section{Single-Molecule FRET Measurements}

All oligonucleotides where purchases at a concentration of $100 \mu \mathrm{M}$ from IDT in nuclease- and salt-free buffer for the single-molecule FRET measurements. For assembling of the aDBB, the oligonucleotides were mixed according to Table S2 in aliquots of $20 \mu \mathrm{L}$ in a solution containing 12.5 $\mathrm{mM} \mathrm{MgCl} 2$ buffered with 10x TAE. The sample solution was subjected to the thermal protocol summarized above. Finally, the mixture was purified with a 4\% 1x TBE agarose gel, which was run for $30 \mathrm{~min}$ at $160 \mathrm{~V}$ in the same buffer. The slowest band (formed by the desired product) was cut out and the structure was extracted by squeezing the cut-out between cover slides. Prior to the single-molecule FRET measurements, the samples were diluted in 1x PBS to achieve a concentration of $100 \mathrm{pM}$. The displacement reaction was performed by adding $\mathrm{D}$ at a 
concentration of $8 \mu \mathrm{M}$. The single-molecule FRET experiments by pulsed interleaved excitation $(\mathrm{PIE})^{47}$ were carried out with a custom-built confocal microscope. To this end, the DNA was placed in custom-built $60 \mu \mathrm{L}$ imaging chambers. The fluorescent donor molecules were excited by a pulsed diode laser (LDH-P- FA-530B, PicoQuant, Germany), at $532 \mathrm{~nm}$ operated with a $20 \mathrm{MHz}$ repetition rate. The excitation intensity was adjusted to $30 \mu \mathrm{W}$. The fluorescent acceptor molecules were excited by a pulsed diode laser (LDH-D-C-640, PicoQuant), at $639 \mathrm{~nm}$ operated with a 20 $\mathrm{MHz}$ repetition rate. The excitation intensity of the sample was adjusted to $30 \mu \mathrm{W}$. The laser pulses were separated by 35 ns by a multichannel picosecond diode laser driver (PDL 828 "Sepia II", PicoQuant) with an oscillator module (SOM 828, PicoQuant). The lasers were coupled into a single mode fiber (P3-488PM-FC, Thorlabs, USA) to obtain a Gaussian beam profile and overlaying laser beams. Circular polarized light was obtained by a linear polarizer (LPVISE100A, Thorlabs) and a quarter-wave plate (AQWP05M- 600, Thorlabs). The laser light was guided into the epi-illuminated confocal microscope (Olympus IX71, Olympus, Japan) by dual-edge beam splitter (z532/633, AHF Analysentechnik AG, Germany) focused by an oil immersion objective (UPLSAPO100XO, NA 1.40, Olympus). The emitted fluorescence was collected through the objective and spatially filtered using a pinhole with $50 \mu \mathrm{m}$ diameter and spectrally split into donor and acceptor channel by a single-edge dichroic mirror (640DCXR, AHF Analysentechnik AG). Fluorescence emission was filtered (donor: Brightline HC582/75 (AHF Analysentechnik AG) and RazorEdge LP 532 (Laser 2000, Germany); acceptor: Shortpass 750 (AHF Analysentechnik AG) and RazorEdge LP 647 (Laser 2000)) and focused on avalanche photodiodes (SPCM-AQRH-14TR, Excelitas Technoligies, USA). The detector outputs were recorded by a time-correlated single photon counting module (HydraHarp 400, PicoQuant). The setup was controlled by a commercial software package (SymPhoTime64, Picoquant). Data analysis was performed using the "PAM" 
software package as described by Schrimpf et al. ${ }^{48}$ Single-molecule events were identified using a two channel APBS-algorithm with a threshold of 10 photons per time window of $500 \mu \mathrm{sec}$ and a minimum photon count of 30 . Gamma correction was performed using the protocol published by Hellenkamp et al. ${ }^{37}$ To remove donor or acceptor only events, the ALEX-2CDE filter was applied using an upper threshold of $15 .^{49}$

\section{Fabrication of DNA-Liposome Hybrid Carriers}

LUVs were prepared by extrusion of a $2 \mathrm{mM}$ 1-palmitoyl- 2-oleoyl-sn-glycero-3phosphocholine (POPC, purchased as powder from Sigma-Aldrich and stored dissolved in chloroform) lipid suspension. The lipids were suspended in 1x PBS, sonicated, and extruded through a $200 \mathrm{~nm}$ pore size membrane.

The coated liposomes were prepared in aliquots of $100 \mu \mathrm{L} .50 \mu \mathrm{L}$ of the LUVs were incubated with approximately $1 \mu \mathrm{M}$ of the aDBB (pre-annealed with the cholesterol-labelled linker) overnight at room temperature (diluting the POPC lipids to $1.2 \mathrm{mM}$ ), rendering $\mathrm{VL}_{\mathrm{Hs}}$. Subsequently, the triskelion was added to obtain a final concentration of approximately $550 \mathrm{nM}$ and incubated with $\mathrm{VL}$ at $4{ }^{\circ} \mathrm{C}$ for $50 \mathrm{~min}\left(\mathrm{VL}_{\mathrm{HS}}\right.$ pre-tempered). This led to a dilution of the linker to approximately $830 \mathrm{nM}$. Due to the addition of the DNA, the liposomes were effectively diluted by half.

\section{Characterization by Dynamic Light Scattering and $\zeta$-Potential Measurements}

Hydrodynamic diameters and $\zeta$-potentials were measured with a ZetaSizer Nano ZSP by Malvern Panalytics. All samples were measured in disposable cuvettes at a final lipid 
concentration of $1 \mathrm{mM}$ in PBS. To measure the $\zeta$-potential, the samples were additionally diluted 1:8 in PBS.

\section{Confocal Microscopy and FRAP Measurements}

Confocal microscopy was performed to assess the ability of the DNA structures to coat and deform the membranes of GUVs. Thus, GUVs (including 200:1 (w/w) NBD-labelled PC) were generated by electroformation, using the protocol described in our previous study. ${ }^{34}$ The linker was annealed with the active hairpin (pre-assembled with the spacer strand as described above) and subsequently added at a concentration of $200 \mathrm{nM}$ and incubated for 2 hours. Finally, the triskelion was added at room temperature and incubated by another hour $(2 \mu \mathrm{L}$ of $6 \mu \mathrm{M} \mathrm{T})$. The spacer strand was purchased with a 5'-ATTO550 modification from IDT. All three arms of the triskelion were labelled with an ATTO647N fluorophore at the 5'-terminal (purchased by IDT). Imaging was conducted using an Olympus F1200 microscope and a 60X oil immersion objective. The samples were illuminated with a $488 \mathrm{~nm}$ (NBD-labelled PC lipids to visualize the lipid membranes), 535 $\mathrm{nm}$ (ATTO550-labelled spacer), and $635 \mathrm{~nm}$ (ATTO647N-labelled triskelion) laser in linesequential acquisition. The coated GUVs were treated with the displacement strand $\mathrm{C}$ for 3 hours to remove the spacer strand and allow the hairpin to close in order to achieve deformation of the vesicles. Before and after this displacement reaction, FRAP analysis was performed to evaluate the diffusion properties of the spacer and triskelion (as an indicator of the extend of DNA polymerization) by bleaching a $3 \mu \mathrm{m}$ large area with the $535 \mathrm{~nm}$ and $635 \mathrm{~nm}$ lasers into the DNA coats. The hyperosmotic pressure was induced by performing the displacement reaction in a solution with an approximately $10 \%$ higher osmolarity (by adjusting the glucose concentration). 


\section{Calcein Release Experiments}

Evidence whether the DNA contraction triggered the release of molecules from the coated liposomes was provided by measuring the leakage of entrapped calcein. POPC lipid films were rehydrated (day 1) in a $60 \mathrm{mM}$ calcein solution diluted in $75 \mathrm{mM} \mathrm{Na}_{2} \mathrm{HPO}_{4}(\mathrm{pH}=7.4)$. The LUVs were purified from free dye by gel filtration using Sephadex G50. Subsequently, the DNA was added as described in the section above, outlining the DLS experiments. The modified linker in combination with the active hairpin $\left(\mathrm{VL}_{\mathrm{HS}}\right)$ was added at day 1 , as well as a version with the inactive hairpin, by omitting $\mathrm{S}\left(\mathrm{VL}_{\mathrm{H}}\right)$. On day 2 , the $\mathrm{T} 1$ triskelion was added to both $\mathrm{VL}_{\mathrm{HS}}$ and $\mathrm{VL}_{\mathrm{H}}$

and incubated for 1 hour at $4{ }^{\circ} \mathrm{C}$. Finally, the displacement strand $\mathrm{S}$ was added to all samples (to account for dilution effects in the non-trigger-responsive samples), and the fluorescence intensity was recorded over time with a ClarioStar Plus plate reader (BMG Labtech, Germany) excitation wavelength $488 \mathrm{~nm} \pm 15 \mathrm{~nm}$, emission recorded at $515 \mathrm{~nm} \pm 20 \mathrm{~nm}$ ) at room temperature. As a control, $\mathrm{C}_{\mathrm{c}}$ was added (altered sequence to prevent hybridization with the spacer strand $\mathrm{S}$ ). At the end of the acquisition time, $1 \%$ of Triton $\mathrm{X}-100$ was added to disrupt the vesicles and record the maximal achievable fluorescence intensity.

\section{Incubation of the DNA-Liposome Hybrid Carriers with HEK293T Cells}

The coated liposomes were fabricated to encapsulate doxorubicin (DOX) and incubated with HEK293T cells. DOX is a widely used anti-cancer drug and imposes toxicity towards cells by inducing DNA strand breaks. ${ }^{50,51}$ Coated liposomes were prepared by rehydrating POPC lipids in a solution of $5 \mathrm{mg} / \mathrm{mL}$ DOX in sterile 1x PBS. DOX was purchased as powder from Stratech Scientific, UK. The remaining fabrication steps followed the protocol summarized above for calcein. 2500 cells were seeded per well of a 96 well plate and covered by $100 \mu \mathrm{L}$ DMEM 
supplemented with $10 \%$ fetal bovine serum and glutamax. The cells were environmentally controlled at $37{ }^{\circ} \mathrm{C}$ and $5 \% \mathrm{CO}_{2}$ incubated for three days to allow adherence and confluence. On the third day, the incubation with the coated liposomes was performed. Alongside the $\mathrm{VL}_{\mathrm{HS}} \mathrm{T}$ carriers, the previously established VLT (all prepared with entrapped DOX) was incubated. For each sample, three wells were prepared for incubation by adding $90 \mu \mathrm{L}$ of sample solution per well for approximately $30 \mathrm{~min}$. Afterwards, $5 \mu \mathrm{L}$ of the displacement strand $\mathrm{D}$, present in a $30 \mu \mathrm{M}$ solution in 1x PBS were added. To three separate wells, $95 \mu \mathrm{L}$ of 1x PBS only was added as a nontoxic control. After an incubation time of approximately 3.5 hours, the supernatant was removed from each well and $30 \mu \mathrm{L}$ of trypsin solution was added to detach the cells. After 2 min of trypsination, the reaction was blocked by adding $100 \mu \mathrm{L}$ of fresh culture medium. The cells were then transferred into centrifugation tubes and spun down for $5 \mathrm{~min}$ at $300 \mathrm{rcf}$. Finally, the supernatant was removed and the cell pellets resuspended in 1x PBS and counted using an automated cell counter (Countess, Thermo Scientific, USA) to adjust for deviating cell numbers for the subsequent viability assay. To assess the viability of the HEK293T cells after the treatment, an ATP-sensitive luciferase bioluminescence assay was performed with CellTiter Glo (Promega, USA). To perform the assay, $100 \mu \mathrm{L}$ of the cells in 1x PBS were pipetted into wells of a black 96well plate (Greiner, Austria) at an approximate concentration of 10000 per $\mu \mathrm{L}$. $30 \mu \mathrm{L}$ of the luciferase buffer were added to each well and incubated for $10 \mathrm{~min}$ at $37^{\circ} \mathrm{C}$. The luminescence emission was analyzed using a ClarioStar Plus plate reader. 


\section{FIGURES}

a

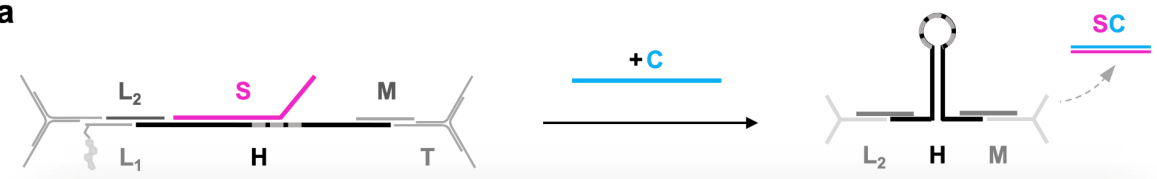

b

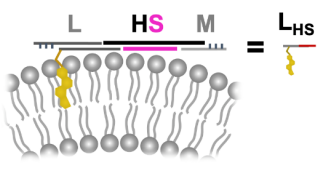

C

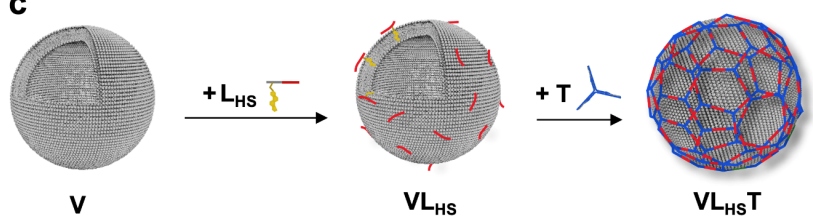

Figure 1. Assembly principle of the active DNA building block and the trigger-responsive DNAliposomal hybrid nanocarrier. (a) Trigger mechanism of the aDBB. The building block comprises a hairpin $\mathrm{H}$ and a pre-annealed, partially complementary sequence $\mathrm{S}$. Mediated by a toehold at the 5'-terminal of S, a complementary trigger strand $\mathrm{C}$ hybridizes with $\mathrm{S}$, allowing $\mathrm{H}$ to close. This leads to a contraction of the two opposite ends. (b) The aDBB is first annealed with a cholesterolTEG-modified linker $\mathrm{L}$ (comprised of $\mathrm{L}_{2}$ and the cholesterol-TEG-labelled $\mathrm{L}_{1}$ ) and a connecting strand $\mathrm{M}$, to render $\mathrm{L}_{\mathrm{Hs}}$. (c) $\mathrm{L}_{\mathrm{HS}}$ is incubated with large unilamellar POPC vesicles $(\mathrm{V})$ and is anchored to the lipid membranes via the cholesterol-TEG modification (rendering $\mathrm{VL}_{\mathrm{HS}}$ ). This allows for polymerization of a triskelion $\mathrm{T}$ which is added in the subsequent step on the surface of the vesicles and hybridizes with $\mathrm{M}$ and $\mathrm{L}_{1}$ (resulting in the final structure $\mathrm{VL}_{\mathrm{HS}} \mathrm{T}$ ). 

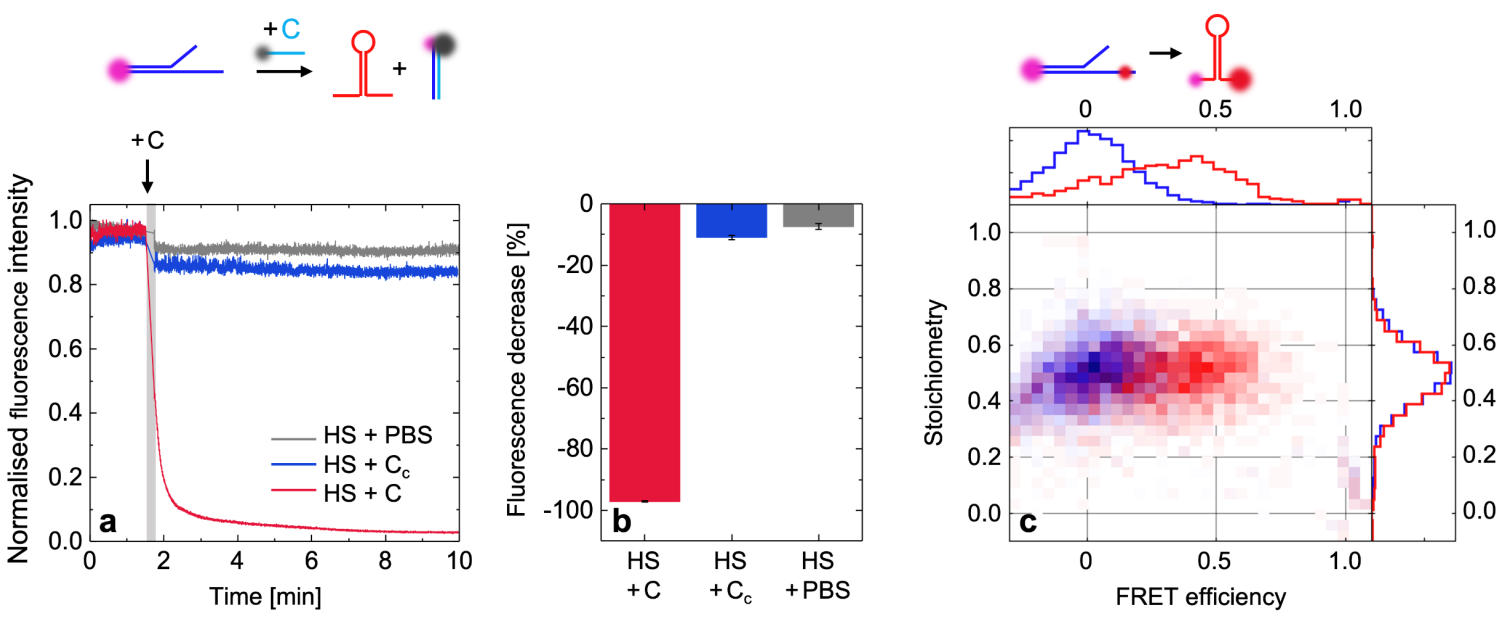

Figure 2. Fluorescence-based assessment of the contraction of the active DNA building block. (a) The addition of $\mathrm{C}$ to the $\mathrm{aDBB}$ in solution leads to a steep drop of the fluorescence intensity, indicating high quenching efficiencies of the fluorophore placed at the 3'-terminal of $\mathrm{S}$ by the quencher placed at the 5'-terminal of $\mathrm{C}$. (b) The strong relative fluorescence decrease suggests successful displacement of $\mathrm{S}$. A control sequence $\mathrm{C}_{\mathrm{c}}$ leads to weak unspecific quenching, comparable to the effect caused by dilution with PBS (error bars represent the standard deviation, $n=3$ ). (c) Single-molecule FRET measurements support the assumption that the displacement of $\mathrm{S}$ results in the contraction of the opposite ends of $\mathrm{H}$ due to self-hybridization. This is indicated by an increase of the FRET efficiency between a donor-acceptor pair placed at the two opposite ends of $\mathrm{H}$. 

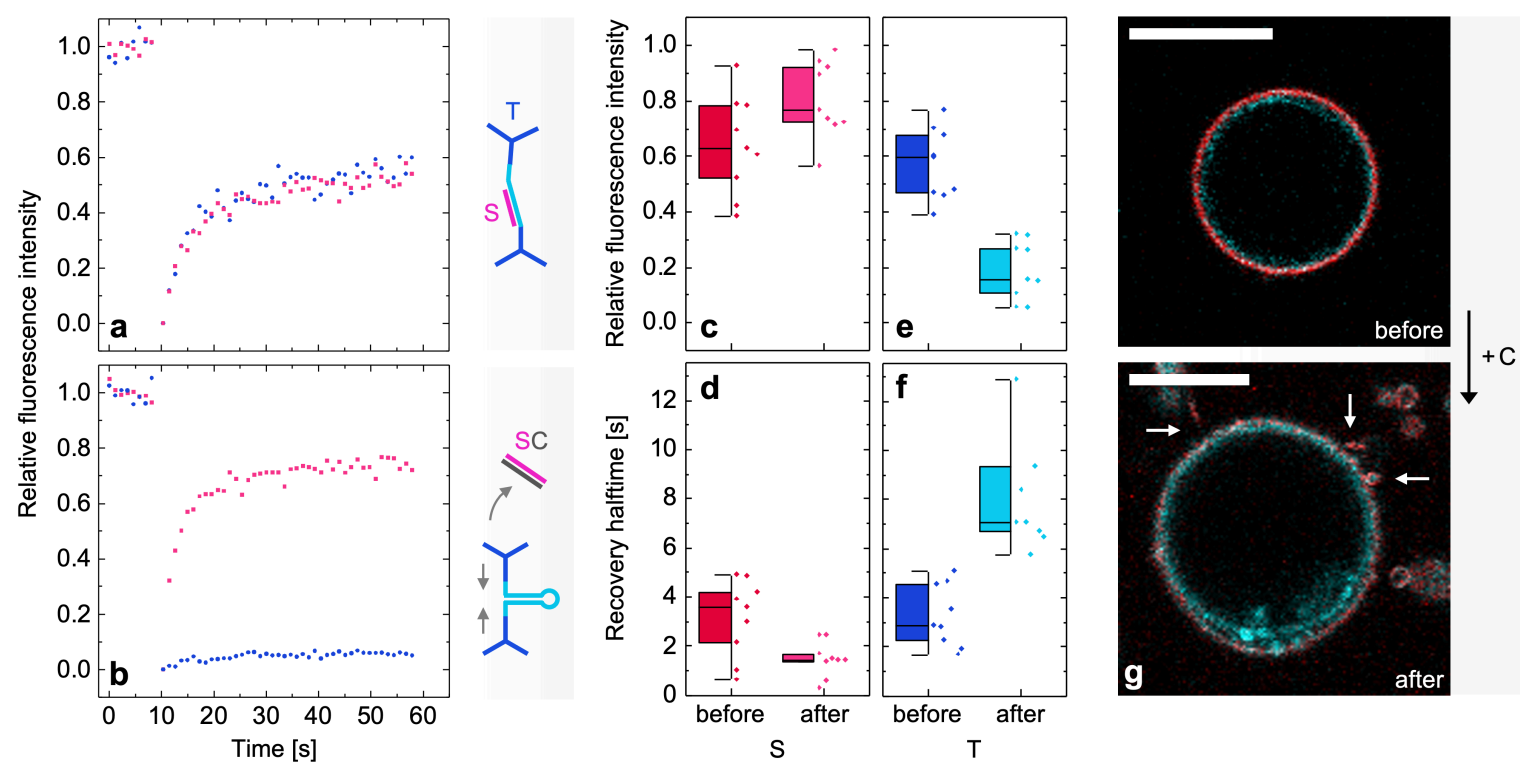

Figure 3. Characterization of DNA-coated GUVs by confocal microscopy and FRAP. (a) Representative FRAP traces recorded on GUVs before the addition of C. S was labelled with ATTO550 (pink data), and $\mathrm{T}$ with ATTO647N (blue data). Both species exhibit similar fluorescence recovery dynamics (described by the recovered fluorescence intensities and recovery halftimes obtained from exponential fitting curves, $n=8$ ). (b) After the addition of $\mathrm{C}$, the fluorescence recovery kinetics of S and T split into two populations: $\mathrm{S}$ is characterized by increased mobility, while $\mathrm{T}$ remains mostly static: the recovered relative fluorescence intensity (c) and the recovery halftime (d) of $\mathrm{S}$ is slightly higher. By contrast, a drop of the levels of recovered fluorescence (e) and slow recovery kinetics (f) can be observed for T (box plots show the upper and lower quartiles, as well as the mean, $\mathrm{n}=8)$. $(\mathrm{g})$ In the presence of a hyperosmotic pressure, visible deformation and tubulation (see arrows) of the coated GUVs could be observed after the displacement of S had been performed (cyan: NBD-labelled PC lipids, red: ATTO647N-labelled T). Scale bars: $10 \mu \mathrm{m}$. 

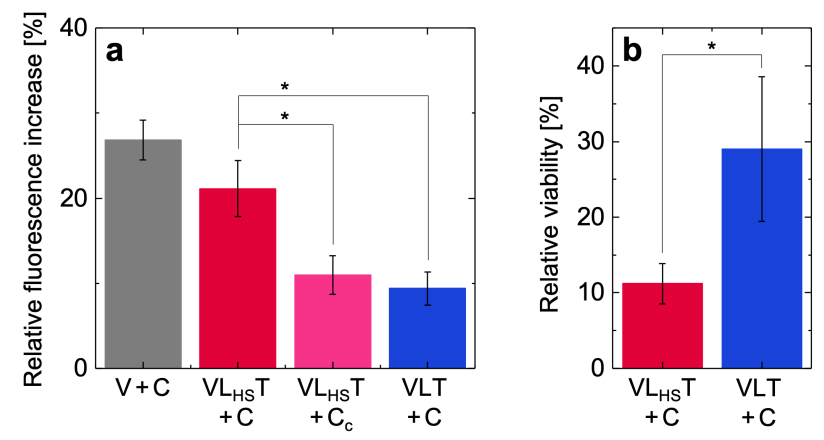

Figure 4. Triggered release of calcein and doxorubicin. (a) The addition of $\mathrm{C}$ to $\mathrm{VL}_{\mathrm{HS}} \mathrm{T}$ induces elevated permeability of the carriers to entrapped calcein $\left(\mathrm{VL}_{\mathrm{HS}} \mathrm{T}+\mathrm{C}\right)$, close to uncoated POPC vesicles $(\mathrm{V}+\mathrm{C})$, whereas an inactive version $(\mathrm{VLT}+\mathrm{C})$ retains calcein to a larger degree. In comparison to $\mathrm{VL}_{\mathrm{HS}} \mathrm{T}+\mathrm{C}$, the calcein release was significantly lower $(\mathrm{p}=0.0060$, $\mathrm{df}=4, \mathrm{t}=$ 5.3227), similar to the addition of a non-hybridizing $\mathrm{C}_{\mathrm{c}}$ to $\mathrm{VL}_{\mathrm{HS}} \mathrm{T}(\mathrm{p}=0.0118$, $\mathrm{df}=4, \mathrm{t}=4.3926)$. (b) DOX is released from the active carrier upon addition of $\mathrm{C}\left(\mathrm{VL}_{\mathrm{HS}} \mathrm{T}+\mathrm{C}\right)$, provoking cytotoxicity in a HEK293T cell culture. In comparison to VLT, the cytotoxic response is statistically significantly increased $(\mathrm{p}=0.0358, \mathrm{df}=4, \mathrm{t}=3.1106)$. Error bars represent the standard deviation $(\mathrm{n}=3)$, the asterisk highlights statistically significant results $(\mathrm{p}<0.05)$. 


\section{AUTHOR INFORMATION}

\section{Corresponding Authors}

* Tuomas P. J. Knowles - Yusuf Hamied Department of Chemistry, University of Cambridge, Lensfield Road, Cambridge CB2 1EW, United Kingdom; Email: tpjk2@cam.ac.uk

* Silvia Hernández-Ainsa - Instituto de Nanociencia y Materiales de Aragón, University of Zaragoza-CSIC, Zaragoza 50009, Spain; ARAID Foundation, Government of Aragon, Zaragoza 50018, Spain; Email: silviamh83@unizar.es

\section{Present Addresses}

$\dagger$ Department of Biosystems Science and Engineering, ETH Zürich, Mattenstrasse 26, 4058 Basel, Switzerland

‡ Feinberg School of Medicine, Northwestern University, 633 Clark St, Evanston, IL 60208, United States

$\S$ School of Engineering, EPFL, 1015 Lausanne, Switzerland

\section{Author Contributions}

The manuscript was written through the contribution of all authors. All authors have given approval to the final version of the manuscript. 


\section{ACKNOWLEDGMENT}

The research leading to these results has received funding from the European Research Council under the European Union's Seventh Framework Programme (FP7/2007-2013) through the ERC grant PhysProt (Agreement No. 337969). K.N.B., P.S.C., and T.P.J.K. are grateful for financial support from the Biotechnology and Biological Sciences Research Council (BBSRC), the Newman Foundation, the Wellcome Trust and the Cambridge Centre for Misfolding Diseases. D.M. is supported by the Winton Programme for the Physics of Sustainability, as well as the Engineering and Physical Sciences Research Council (EPSRC). T.S. and P.T. gratefully acknowledge funding by the Bavarian Ministry of Science and the Arts through the ONE MUNICH Project "Munich Multiscale Biofabrication" and by the LMU-Cambridge strategic partnership. S.H.A. acknowledges funding by the Gobierno de Aragón-FSE (Research Group E47_20R). The authors would like to thank Prof. Ulrich Keyser, Dr. Florian Buhr, and Anne Jacobs for insightful discussions. 


\section{REFERENCES}

(1) Senapati, S.; Mahanta, A. K.; Kumar, S.; Maiti, P. Controlled Drug Delivery Vehicles for Cancer Treatment and Their Performance. Signal Transduct. Target. Ther. 2018, 3 (1), 119. https://doi.org/10.1038/s41392-017-0004-3.

(2) Coelho, J. F.; Ferreira, P. C.; Alves, P.; Cordeiro, R.; Fonseca, A. C.; Góis, J. R.; Gil, M. H. Drug Delivery Systems: Advanced Technologies Potentially Applicable in Personalized Treatments. EPMA J. 2010, 1 (1), 164-209. https://doi.org/10.1007/s13167-010-0001-x.

(3) Kumar, V.; Palazzolo, S.; Bayda, S.; Corona, G.; Toffoli, G.; Rizzolio, F. DNA Nanotechnology for Cancer Therapy. Theranostics 2016, 6 (5), 710-725. https://doi.org/10.7150/thno.14203.

(4) Joseph, J.; Baumann, K. N.; Postigo, A.; Bollepalli, L.; Bohndiek, S. E.; Hernández-Ainsa, S. DNA-Based Nanocarriers to Enhance the Optoacoustic Contrast of Tumors In Vivo. Adv. Healthc. Mater. 2021, 10 (2), 2001739. https://doi.org/10.1002/adhm.202001739.

(5) Petschauer, J. S.; Madden, A. J.; Kirschbrown, W. P.; Song, G.; Zamboni, W. C. The Effects of Nanoparticle Drug Loading on the Pharmacokinetics of Anticancer Agents. Nanomedicine 2015, 10 (3), 447-463. https://doi.org/10.2217/nnm.14.179.

(6) De Leo, V.; Milano, F.; Agostiano, A.; Catucci, L. Recent Advancements in Polymer/Liposome Assembly for Drug Delivery: From Surface Modifications to Hybrid Vesicles. Polymers (Basel). 2021, 13 (7), 1027. https://doi.org/10.3390/polym13071027.

(7) Loomis, K.; McNeeley, K.; Bellamkonda, R. V. Nanoparticles with Targeting, Triggered Release, and Imaging Functionality for Cancer Applications. Soft Matter 2011, 7 (3), 839_ 
856. https://doi.org/10.1039/c0sm00534g.

(8) Fleige, E.; Quadir, M. A.; Haag, R. Stimuli-Responsive Polymeric Nanocarriers for the Controlled Transport of Active Compounds: Concepts and Applications. Adv. Drug Deliv. Rev. 2012, 64 (9), 866-884. https://doi.org/10.1016/J.ADDR.2012.01.020.

(9) Lee, J. H.; Yeo, Y. Controlled Drug Release from Pharmaceutical Nanocarriers. Chem. Eng. Sci. 2015, 125, 75-84. https://doi.org/10.1016/j.ces.2014.08.046.

(10) Weber, J.; Beard, P. C.; Bohndiek, S. E. Contrast Agents for Molecular Photoacoustic Imaging. Nat. Methods 2016, 13 (8), 639-650. https://doi.org/10.1038/nmeth.3929.

(11) Fujimoto, J. G.; Schmitt, J.; Swanson, E.; Aguirre, A. D.; Jang, I.-K. The Development of Optical Coherence Tomography. In Cardiovascular OCT Imaging; Springer International Publishing, 2020; pp 1-23. https://doi.org/10.1007/978-3-030-25711-8_1.

(12) Zhao, W.; Zhao, Y.; Wang, Q.; Liu, T.; Sun, J.; Zhang, R. Remote Light-Responsive Nanocarriers for Controlled Drug Delivery: Advances and Perspectives. Small 2019, 15 (45), 1903060. https://doi.org/10.1002/smll.201903060.

(13) Deng, W.; Chen, W.; Clement, S.; Guller, A.; Zhao, Z.; Engel, A.; Goldys, E. M. Controlled Gene and Drug Release from a Liposomal Delivery Platform Triggered by X-Ray Radiation. Nat. Commun. 2018, 9 (1), 1-11. https://doi.org/10.1038/s41467-018-05118-3.

(14) Decuzzi, P.; Cook, A. B. Harnessing Endogenous Stimuli for Responsive Materials in $\begin{array}{llllll}\text { Theranostics. } & \text { ACS } & \text { Nano } & \text { 2021, } & 15 & \text { (2), }\end{array}$ https://doi.org/10.1021/acsnano.0c09115. 
(15) Chen, L.; Zhang, J.; Lin, Z.; Zhang, Z.; Mao, M.; Wu, J.; Li, Q.; Zhang, Y.; Fan, C. Pharmaceutical Applications of Framework Nucleic Acids. Acta Pharm. Sin. B 2021. https://doi.org/10.1016/j.apsb.2021.05.022.

(16) Douglas, S. M.; Bachelet, I.; Church, G. M. A Logic-Gated Nanorobot for Targeted Transport of Molecular Payloads. Science 2012, 335 (6070), 831-834. https://doi.org/10.1126/science.1214081.

(17) Andersen, E. S.; Dong, M.; Nielsen, M. M.; Jahn, K.; Subramani, R.; Mamdouh, W.; Golas, M. M.; Sander, B.; Stark, H.; Oliveira, C. L. P.; Pedersen, J. S.; Birkedal, V.; Besenbacher, F.; Gothelf, K. V.; Kjems, J. Self-Assembly of a Nanoscale DNA Box with a Controllable Lid. Nature 2009, 459 (7243), 73-76. https://doi.org/10.1038/nature07971.

(18) Madhanagopal, B. R.; Zhang, S.; Demirel, E.; Wady, H.; Chandrasekaran, A. R. DNA Nanocarriers: Programmed to Deliver. Trends Biochem. Sci. 2018, 43 (12), 997-1013. https://doi.org/10.1016/J.TIBS.2018.09.010.

(19) Liphardt, J.; Onoa, B.; Smith, S. B.; Jr, T. I.; Bustamante, C. Reversible Unfolding of Single RNA Molecules by Mechanical Force. Science 2001, 292 (5517), 733-737. https://doi.org/10.1126/science.1058498.

(20) Bercy, M.; Bockelmann, U. Hairpins under Tension: RNA versus DNA. Nucleic Acids Res. 2015, 43 (20), 9928-9936. https://doi.org/10.1093/nar/gkv860.

(21) Strunz, T.; Oroszlan, K.; Schäfer, R.; Güntherodt, H. J. Dynamic Force Spectroscopy of Single DNA Molecules. Proc. Natl. Acad. Sci. U. S. A. 1999, 96 (20), 11277-11282. https://doi.org/10.1073/pnas.96.20.11277. 
(22) Gambari, R.; Brognara, E.; Spandidos, D. A.; Fabbri, E. Targeting OncomiRNAs and Mimicking Tumor Suppressor MiRNAs: New Trends in the Development of MiRNA Therapeutic Strategies in Oncology (Review). Int. J. Oncol. 2016, 49 (1), 5-32. https://doi.org/10.3892/ijo.2016.3503.

(23) He, H.; Tian, D.; Guo, J.; Liu, M.; Chen, Z.; Hamdy, F. C.; Helleday, T.; Su, M.; Ying, S. DNA Damage Response in Peritumoral Regions of Oesophageal Cancer Microenvironment. $\quad$ Carcinogenesis $\quad$ 2013, $34 \quad$ (1), 139-145. https://doi.org/10.1093/carcin/bgs301.

(24) Piwecka, M.; Rolle, K.; Belter, A.; Barciszewska, A. M.; Zywicki, M.; Michalak, M.; Nowak, S.; Naskret-Barciszewska, M. Z.; Barciszewski, J. Comprehensive Analysis of MicroRNA Expression Profile in Malignant Glioma Tissues. Mol. Oncol. 2015, 9 (7), 13241340. https://doi.org/10.1016/j.molonc.2015.03.007.

(25) Sanguanini, M.; Baumann, K. N.; Preet, S.; Chia, S.; Habchi, J.; Knowles, T. P. J.; Vendruscolo, M. Complexity in Lipid Membrane Composition Induces Resilience to A 342 Aggregation. ACS Chem. Neurosci. 2020, $11 \quad$ (9), 1347-1352. https://doi.org/10.1021/acschemneuro.0c00101.

(26) Guimarães, D.; Cavaco-Paulo, A.; Nogueira, E. Design of Liposomes as Drug Delivery System for Therapeutic Applications. Int. J. Pharm. 2021, 601, 120571. https://doi.org/10.1016/J.IJPHARM.2021.120571.

(27) Ohmann, A.; Li, C.-Y.; Maffeo, C.; Al Nahas, K.; Baumann, K. N.; Göpfrich, K.; Yoo, J.; Keyser, U. F.; Aksimentiev, A. A Synthetic Enzyme Built from DNA Flips 107 Lipids per Second in Biological Membranes. Nat. Commun. 2018, 9 (1), 2426. 
https://doi.org/10.1038/s41467-018-04821-5.

(28) Burns, J. R.; Göpfrich, K.; Wood, J. W.; Thacker, V. V.; Stulz, E.; Keyser, U. F.; Howorka, S. Lipid-Bilayer-Spanning DNA Nanopores with a Bifunctional Porphyrin Anchor. Angew. Chemie - Int. Ed. 2013, 52 (46), 12069-12072. https://doi.org/10.1002/anie.201305765.

(29) Hernández-Ainsa, S.; Ricci, M.; Hilton, L.; Aviñó, A.; Eritja, R.; Keyser, U. F. Controlling the Reversible Assembly of Liposomes through a Multistimuli Responsive Anchored DNA. Nano Lett. 2016, 16 (7), 4462-4466. https://doi.org/10.1021/acs.nanolett.6b01618.

(30) Göpfrich, K.; Zettl, T.; Meijering, A. E. C.; Hernández-Ainsa, S.; Kocabey, S.; Liedl, T.; Keyser, U. F. DNA-Tile Structures Induce Ionic Currents through Lipid Membranes. Nano Lett. 2015, 15 (5), 3134-3138. https://doi.org/10.1021/acs.nanolett.5b00189.

(31) Czogalla, A.; Franquelim, H. G.; Schwille, P. DNA Nanostructures on Membranes as Tools for Synthetic Biology. Biophys. J. 2016, 110 (8), 1698-1707. https://doi.org/10.1016/J.BPJ.2016.03.015.

(32) Franquelim, H. G.; Khmelinskaia, A.; Sobczak, J.-P.; Dietz, H.; Schwille, P. Membrane Sculpting by Curved DNA Origami Scaffolds. Nat. Commun. 2018, 9 (1), 811. https://doi.org/10.1038/s41467-018-03198-9.

(33) Kocabey, S.; Kempter, S.; List, J.; Xing, Y.; Bae, W.; Schiffels, D.; Shih, W. M.; Simmel, F. C.; Liedl, T. Membrane-Assisted Growth of DNA Origami Nanostructure Arrays. ACS Nano 2015, 9 (4), 3530-3539. https://doi.org/10.1021/acsnano.5b00161.

(34) Baumann, K. N.; Piantanida, L.; García-Nafría, J.; Sobota, D.; Voïtchovsky, K.; Knowles, T. P. J.; Hernández-Ainsa, S. Coating and Stabilization of Liposomes by Clathrin-Inspired 
DNA Self-Assembly. ACS Nano $\quad$ 2020, $14 \quad$ (2), 2316-2323. https://doi.org/10.1021/acsnano.9b09453.

(35) Ishii, Y.; Yoshida, T.; Funatsu, T.; Wazawa, T.; Yanagida, T. Fluorescence Resonance Energy Transfer between Single Fluorophores Attached to a Coiled-Coil Protein in Aqueous Solution. Chem. Phys. 1999, 247 (1), 163-173. https://doi.org/10.1016/S03010104(99)00174-3.

(36) Forster, T. Energiewanderung Und Fluoreszenz. Naturwissenschaften 1946, 33 (6), 166175. https://doi.org/10.1007/BF00585226.

(37) Hellenkamp, B.; Schmid, S.; Doroshenko, O.; Opanasyuk, O.; Kühnemuth, R.; Rezaei Adariani, S.; Ambrose, B.; Aznauryan, M.; Barth, A.; Birkedal, V.; Bowen, M. E.; Chen, H.; Cordes, T.; Eilert, T.; Fijen, C.; Gebhardt, C.; Götz, M.; Gouridis, G.; Gratton, E.; Ha, T.; Hao, P.; Hanke, C. A.; Hartmann, A.; Hendrix, J.; Hildebrandt, L. L.; Hirschfeld, V.; Hohlbein, J.; Hua, B.; Hübner, C. G.; Kallis, E.; Kapanidis, A. N.; Kim, J.-Y.; Krainer, G.; Lamb, D. C.; Lee, N. K.; Lemke, E. A.; Levesque, B.; Levitus, M.; McCann, J. J.; NarediRainer, N.; Nettels, D.; Ngo, T.; Qiu, R.; Robb, N. C.; Röcker, C.; Sanabria, H.; Schlierf, M.; Schröder, T.; Schuler, B.; Seidel, H.; Streit, L.; Thurn, J.; Tinnefeld, P.; Tyagi, S.; Vandenberk, N.; Vera, A. M.; Weninger, K. R.; Wünsch, B.; Yanez-Orozco, I. S.; Michaelis, J.; Seidel, C. A. M.; Craggs, T. D.; Hugel, T. Precision and Accuracy of SingleMolecule FRET Measurements - a Multi-Laboratory Benchmark Study. Nat. Methods 2018, 15 (9), 669-676. https://doi.org/10.1038/s41592-018-0085-0.

(38) Morzy, D.; Rubio-Sánchez, R.; Joshi, H.; Aksimentiev, A.; Di Michele, L.; Keyser, U. F. Cations Regulate Membrane Attachment and Functionality of DNA Nanostructures. J. Am. 
Chem. Soc. 2021, 143 (19), 7358-7367. https://doi.org/10.1021/jacs.1c00166.

(39) Zamboni, W. C. Liposomal, Nanoparticle, and Conjugated Formulations of Anticancer Agents. Clin. Cancer Res. 2005, 11 (23), 8230-8234. https://doi.org/10.1158/10780432.CCR-05-1895.

(40) Huang, F.; Liao, W.C.; Sohn, Y. S.; Nechushtai, R.; Lu, C. H.; Willner, I. Light-Responsive and PH-Responsive DNA Microcapsules for Controlled Release of Loads. J. Am. Chem. Soc. 2016, 138 (28), 8936-8945. https://doi.org/10.1021/jacs.6b04773.

(41) Sercombe, L.; Veerati, T.; Moheimani, F.; Wu, S. Y.; Sood, A. K.; Hua, S. Advances and Challenges of Liposome Assisted Drug Delivery. Front. Pharmacol. 2015, 6 (DEC), 286. https://doi.org/10.3389/fphar.2015.00286.

(42) Iqbal, N.; Iqbal, N. Human Epidermal Growth Factor Receptor 2 (HER2) in Cancers: Overexpression and Therapeutic Implications. Mol. Biol. Int. 2014, 2014, 852748. https://doi.org/10.1155/2014/852748.

(43) Sledge, G. W. VEGF-Targeting Therapy for Breast Cancer. J. Mammary Gland Biol. Neoplasia 2005, 10 (4), 319-323. https://doi.org/10.1007/s10911-006-9005-5.

(44) Bunker, A.; Magarkar, A.; Viitala, T. Rational Design of Liposomal Drug Delivery Systems, a Review: Combined Experimental and Computational Studies of Lipid Membranes, Liposomes and Their PEGylation. Biochim. Biophys. Acta - Biomembr. 2016, 1858 (10), 2334-2352. https://doi.org/10.1016/j.bbamem.2016.02.025.

(45) Dore-Savard, L.; Lee, E.; Kakkad, S.; Popel, A. S.; Bhujwalla, Z. M. The Angiogenic Secretome in VEGF Overexpressing Breast Cancer Xenografts. Sci. Rep. 2016, 6 (1), 1-10. 
https://doi.org/10.1038/srep39460.

(46) Mi, P. Stimuli-Responsive Nanocarriers for Drug Delivery, Tumor Imaging, Therapy and $\begin{array}{lllll}\text { Theranostics. } & \text { Theranostics } & \mathbf{2 0 2 0}, & 10 & (10),\end{array}$ https://doi.org/10.7150/THNO.38069.

(47) Müller, B. K.; Zaychikov, E.; Bräuchle, C.; Lamb, D. C. Pulsed Interleaved Excitation. Biophys. J. 2005, 89 (5), 3508-3522. https://doi.org/10.1529/biophysj.105.064766.

(48) Schrimpf, W.; Barth, A.; Hendrix, J.; Lamb, D. C. PAM: A Framework for Integrated Analysis of Imaging, Single-Molecule, and Ensemble Fluorescence Data. Biophys. J. 2018, 114 (7), 1518-1528. https://doi.org/10.1016/j.bpj.2018.02.035.

(49) Tomov, T. E.; Tsukanov, R.; Masoud, R.; Liber, M.; Plavner, N.; Nir, E. Disentangling Subpopulations in Single-Molecule FRET and ALEX Experiments with Photon Distribution Analysis. Biophys. J. 2012, 102 (5), 1163-1173. https://doi.org/10.1016/j.bpj.2011.11.4025.

(50) Lopes De Menezes, D. E.; Kirchmeier, M. J.; Gagne, J. F.; Pilarski, L. M.; Allen, T. M. Cellular Trafficking and Cytotoxicity of Anti-CD19-Targeted Liposomal Doxorubicin in B Lymphoma Cells. J. Liposome Res. 1999, 9 (2), 199-228. https://doi.org/10.3109/08982109909024786.

(51) Chatterjee, K.; Zhang, J.; Honbo, N.; Karliner, J. S. Doxorubicin Cardiomyopathy. Cardiology 2010, 115 (2), 155-162. https://doi.org/10.1159/000265166. 